\title{
Reductionists lay claim to the mind
}

There are deep disagreements about how science should deal with the problem of consciousness. But neuroscientists should soon be in a position to set the agenda. The result is likely to be rapid progress.

Although the object of their study is also the organ that produces consciousness, neurobiologists are often reticent on the topic following their failure so far to reach any agreement about how (if at all) this phenomenon should be studied. But they have recently begun to raise their heads above the parapet, to join equally rash physicists, philosophers, psychologists and computer scientists. This community is united in accepting that consciousness has something to do with the brain (and that neurobiology might therefore be relevant), but in little else.

Perhaps the most basic question is whether existing physical principles are sufficient to explain the brain. The boldest attempt to 'rescue' consciousness from neurobiological reductionism comes famously from Roger Penrose. He argues that the brain performs feats of understanding that cannot be computed and so cannot be implemented by any system based on known physical laws. Instead, he believes, explaining the brain will require a new theory linking events at the quantum level with those of the macroscopic world. Adherents to this view invoke key bits of cellular infrastructure - microtubules - as the most likely substrate for coherent quantum-level phenomena, but most neurobiologists are unimpressed. It remains to be demonstrated that quantal processes might show unusual manifestations in microtubules, and it is difficult to see how they could influence or be influenced by neurotransmitter release or electrical depolarization of neuronal membranes, events with undoubted relevance to mental processes.

Most importantly, there is no direct evidence that current physical concepts are inadequate to explain the brain, or that a quantum-level theory would be any better. Penrose believes that neurobiologists will indeed encounter phenomena that cannot be accounted for by known principles, perhaps even in relatively simple nervous systems. But, unless this happens, neurobiologists might best treat his argument as an obstacle to be side-stepped rather than as a source of illumination. That certainly seemed to be the majority response to his views at a multidisciplinary meeting on this topic ("Towards a science of consciousness") last month in Tucson, Arizona, which provided a snapshot of current debates.

If the brain can be entirely explained in terms of known physical processes, then perhaps consciousness has no independent causal effects. How then was it favoured by natural selection? Perhaps it wasn't. Consciousness may simply be an epiphenomenon of certain arrangements of matter, or indeed of all matter: as one speaker at the conference remarked, perhaps even an atom feels a spasm of relief as an electron falls to a lower orbital. Complex brains would presumably generate more complex forms of consciousness as a by-product of their more sophisticated computations. But this is not very helpful in explaining why (say) the colour red feels the way it does, or in deducing from the neurobiology of echolocation how it feels to be a bat, or in deciding whether it is unethical to unplug a computer. David Chalmers (whose book The Conscious Mind is reviewed on page 123) refers to this class of questions as "the hard problem"; unlike the (relatively) easy problem of explaining how the brain generates behaviour, the experiential aspects of our brain states seem to defy any conceivable explanation in terms of known concepts.

But not everyone accepts this 'hard/soft' distinction; for one thing, it tends to marginalize neuroscience by implying that it can never hope to illuminate the 'hard' problem. Being marginalized, however, does have the advantage of leaving neuroscientists free to tackle the 'easy' problems untroubled by philosophical angst. While the full experiential aspect of consciousness may be impossible to define or measure, an 'easy' definition is both feasible and useful: to be conscious of something is to have a flexible neuronal representation that can be used to drive many different behavioural outputs. In an experimental situation, this can be an arbitrarily specified button press or (for humans) a statement that one is conscious. The subject's testimony can then be correlated with the accompanying neural activity, either by functional brain imaging or (in monkeys trained to report their perceptions) by recording the activities of individual neurons.

So far, most such studies have employed visual stimuli, but other approaches, along with conditions such as sleep, anaesthesia, and perhaps even trance, offer further opportunities to search for neural correlates of conscious states. The underlying assumption is that systematic correlations are there to be found, and that conscious states correspond not to some loosely defined global state of the entire brain, but much more precisely to the activities of specific populations of neurons.

Whatever the nature of these patterns of activity, credit is due to Daniel Dennett for warning against the "Cartesian Theatre" fallacy that perceptual representations converge on a single hypothetical "perceiver". Our current knowledge of the brain gives no support to such a view; visual representations, for instance, are distributed across many brain regions. Similarly, Dennett doubts the existence of any "central meaner" that decides what we mean to say and do. Instead, he envisages that multiple neuronal representations are constantly competing for control of both perception and action. And to those who reply that "it just doesn't feel like that", he rightly warns against the assumption that a few minutes of introspection are sufficient to test a theory of brain function.

Those who still think that their intuitions are a reliable guide to how their minds work should reflect on the condition known as anosognosia. This rare syndrome arises after damage to the right parietal lobe, and leaves patients not only paralysed on the left side of their bodies but also unable to acknowledge the fact. When asked to perform tasks with the paralysed hand, they confabulate, and many will also deny the paralysis of a fellow patient. And as if this were not bizarre enough, the false belief (though not the paralysis) can be temporarily corrected by flushing cold water into the left ear. There is reason to believe that anosognosics have suffered damage to a "belief mechanism" that normally updates our world-view in the face of new information; it should now be possible to identify the brain regions involved.

As neuroscience sheds light on perception, memory, emotion, decision-making and belief, it will become increasingly difficult to deny its central importance in understanding consciousness. Many other approaches will come to seem correspondingly irrelevant. But whether neuroscientists will be able to make their discoveries 'feel' like an explanation for what goes on inside our heads, whether we will ever be able to reconcile their findings with our intuitions about our own mental lives, seems less certain. To meet that challenge, neuroscience may yet be grateful for the philosophers' help. 